\title{
Progress and Prospects in Human Genetic Research into Age-Related Hearing Impairment
}

\author{
Yasue Uchida, ${ }^{1,2}$ Saiko Sugiura, ${ }^{2}$ Michihiko Sone, ${ }^{3}$ \\ Hiromi Ueda, ${ }^{1}$ and Tsutomu Nakashima ${ }^{3}$ \\ ${ }^{1}$ Department of Otolaryngology, Aichi Medical University, Nagakute, Aichi 480-1195, Japan \\ ${ }^{2}$ Department of Otorhinolaryngology, National Center for Geriatrics and Gerontology, Aichi 474-8511, Japan \\ ${ }^{3}$ Department of Otorhinolaryngology, Nagoya University Graduate School of Medicine, Nagoya 466-8550, Japan
}

Correspondence should be addressed to Yasue Uchida; yasueu@aichi-med-u.ac.jp

Received 29 January 2014; Accepted 26 June 2014; Published 22 July 2014

Academic Editor: Johan H. M. Frijns

Copyright (C) 2014 Yasue Uchida et al. This is an open access article distributed under the Creative Commons Attribution License, which permits unrestricted use, distribution, and reproduction in any medium, provided the original work is properly cited.

\begin{abstract}
Age-related hearing impairment (ARHI) is a complex, multifactorial disorder that is attributable to confounding intrinsic and extrinsic factors. The degree of impairment shows substantial variation between individuals, as is also observed in the senescence of other functions. This individual variation would seem to refute the stereotypical view that hearing deterioration with age is inevitable and may indicate that there is ample scope for preventive intervention. Genetic predisposition could account for a sizable proportion of interindividual variation. Over the past decade or so, tremendous progress has been made through research into the genetics of various forms of hearing impairment, including ARHI and our knowledge of the complex mechanisms of auditory function has increased substantially. Here, we give an overview of recent investigations aimed at identifying the genetic risk factors involved in ARHI and of what we currently know about its pathophysiology. This review is divided into the following sections: (i) genes causing monogenic hearing impairment with phenotypic similarities to ARHI; (ii) genes involved in oxidative stress, biologic stress responses, and mitochondrial dysfunction; and (iii) candidate genes for senescence, other geriatric diseases, and neurodegeneration. Progress and prospects in genetic research are discussed.
\end{abstract}

\section{Introduction}

Age-related hearing impairment (ARHI), also known as presbycusis, is the most common sensory impairment among the elderly. Typical features of ARHI are gradual progression later in life and bilaterally symmetrical sensorineural hearing loss which involves the higher frequencies. However, ARHI shows extensive variation, as is also observed in the senescence of other functions. The progression and the severity of hearing impairment vary considerably between individuals. ARHI is a complex, multifactorial trait that is attributable to confounding intrinsic and extrinsic factors. Genetic predisposition could account for a sizable proportion of interindividual variation. Since we published our review on the molecular genetic epidemiology of ARHI in 2011 [1], the number of investigations into the genetics of ARHI has grown hugely. Here, we present the most recent findings in genetic research and discuss the progress of such research to date and future prospects.

This review is divided into the following three areas, although there is likely some overlap between the genes described in each:

(i) genes causing monogenic hearing impairment with phenotypic similarities to ARHI,

(ii) genes involved in oxidative stress, biologic stress responses, and mitochondrial dysfunction,

(iii) candidate genes for senescence, other geriatric diseases, and neurodegeneration.

Recent topic-based genetic contributions, which have been followed by a flurry of additional reports, have been preferentially selected. 


\section{Genes Causing Monogenic Hearing Impairment with Phenotypic Similarities to ARHI}

These genes, which can cause hearing impairment such as pronounced progressive sensorineural hearing loss in the high frequencies, are excellent candidate ARHI susceptibility genes [2]. Many investigators have attempted to verify the association between certain genes causing monogenic nonsyndromic hearing impairment and ARHI. We provide leading examples here and also list them in Table 1.

2.1. DFNA28 = GRHL2/TFCP2L3/BOM (OMIM: 608576). The DFNA28 (OMIM: 608641) locus is occupied by the GRHL2 (grainyhead like 2) gene, which is also known as TFCP2L3 (transcription factor cellular promoter 2-like 3) or $B O M$ (brother of mammalian grainyhead), and is a widely expressed transcription factor in human epithelial tissues [11]. GRHL2 spans approximately $177 \mathrm{~kb}$ on chromosome $8 \mathrm{q} 22.3$ (NCBI 37/hg19), contains 16 exons, and is translated into a 625-amino-acid protein. It was first associated with the DFNA28 locus through mapping studies involving a five-generation North American family affected with mild to moderate postlingual progressive bilateral sensorineural hearing loss [12]. A frameshift mutation in GRHL2 (c.1609_1610insC) that results from a truncation in exon 14 is responsible for DFNA28-associated autosomal dominant hearing loss.

Very recently, a second DFNA28-causing mutation and the first splice-site mutation in GRHL2 (c.1258-1G>A) were reported in a family affected with nonsyndromic hearing loss $[12,13]$. GRHL2 participates in the differentiation and maintenance of epithelial cells throughout life [11]. Impaired epithelial cell integrity is the most reasonable pathologic explanation as to its involvement in late-onset hearing impairment [3, 12]. van Laer et al. have concluded that GRHL2 is an ARHI susceptibility gene from the results of an association study performed with 2418 samples from individuals with ARHI at nine centers in seven European countries [3]. After statistical analysis of 703 single nucleotide polymorphisms (SNPs) selected from 70 candidate genes, they demonstrated that the three top-ranked SNPs all resided in GRHL2, in a region of approximately $16 \mathrm{~kb}$. Particularly, the most significant SNP, rs10955255, was replicated in two of nine independent sample sets; and the odds ratios (ORs) of all nine sample sets had similar implications. However, when Lin et al. tried to replicate these results in a Han Taiwanese population, they found no positive association between the GRHL2 SNP locus (rs10955255: A/G) in intron 1 (coordinate: 102605581) and ARHI [4].

The hearing loss in the family with c.1609-1610insC can be categorized as mild to moderate across all frequencies in the initial stages but progressing toward severe hearing loss of the high frequencies in the fifth decade. The age at onset is variable; the youngest patient was diagnosed in his first decade. DFNA28 hearing loss does not then entirely match the typical features of ARHI. The functional properties of GRHL2 within the inner ear will also critically depend on the presence of its target genes, given that GRHL2 is a transcription factor [3].

2.2. DFNA2 = KCNQ4 (OMIM: 603537). KCNQ4 (potassium voltage-gated channel member 4) encodes a voltage-gated potassium channel [14], which plays a role in potassium recycling in the inner ear and is expressed in hair cells of the cochlea and the vestibular apparatus and in the auditory nuclei of the brainstem [15]. Signal transduction in the cochlea depends on maintenance of a high potassium concentration and a positive potential in the endolymph. This is mediated through ion transport across the stria vascularis into the hair cells and efflux of $\mathrm{K}^{+}$probably through voltagegated ion channels. Mutations in these channels have been thought to implicate candidate genes for individuals with slowly progressive hearing loss.

Mutations in KCNQ4 cause an autosomal-dominant type of nonsyndromic hearing loss, DFNA2. It has been reported that DFNA2-associated hearing loss is typically late in onset, is involved in high-frequency hearing loss, and progresses over time [16]. Although the KCNQ4 gene has 14 exons encoding a protein of 695 amino acids, the mutations seem to cluster around exons 4-7, with missense mutations being the most common [17]. The progressive nature of the hearing loss is a common feature of patients with KCNQ4 mutations, regardless of the particular mutation [18].

van Eyken et al. examined SNPs spread across KCNQ4 for association with ARHI by treating ARHI as a quantitative trait in two independent Caucasian populations. For both populations, several SNPs in a region spanning $13 \mathrm{~kb}$ in the middle of the KCNQ4 gene were significantly associated with ARHI [5].

2.3. DFNA5 = DFNA5 (OMIM: 608798). DFNA5 was originally discovered in a Dutch family with autosomal dominant nonsyndromic hearing impairment [19]. The auditory feature of DFNA5 closely resembles that of the most frequent type of ARHI, namely, sensorineural progressive loss that begins in the high frequencies. van Laer et al. have investigated quantitative trait linkage analysis (a quantitative measure of high-frequency hearing loss) in extended pedigrees as well as in two different association studies involving two amino acid substitution SNPs in DFNA5. They have concluded that no strong association exists between DFNA5 and ARHI, because no significant linkage was detected between ARHI and microsatellite markers from the DFNA5 region and no significant differences were found in genotypes between individuals with good hearing and those with hearing impairment [6].

Different mutations have been found among Korean, Chinese, and Dutch families with DFNA5-associated hearing loss, all exerting a highly specific gain-of-function effect. In these families exon 8 is skipped during splicing, causing a frameshift mutation and premature termination of the protein $[20,21]$. Later, it became clear that the DFNA5 protein acts as a tumor suppressor in several frequent types of cancer and that it can induce apoptosis [22]. 


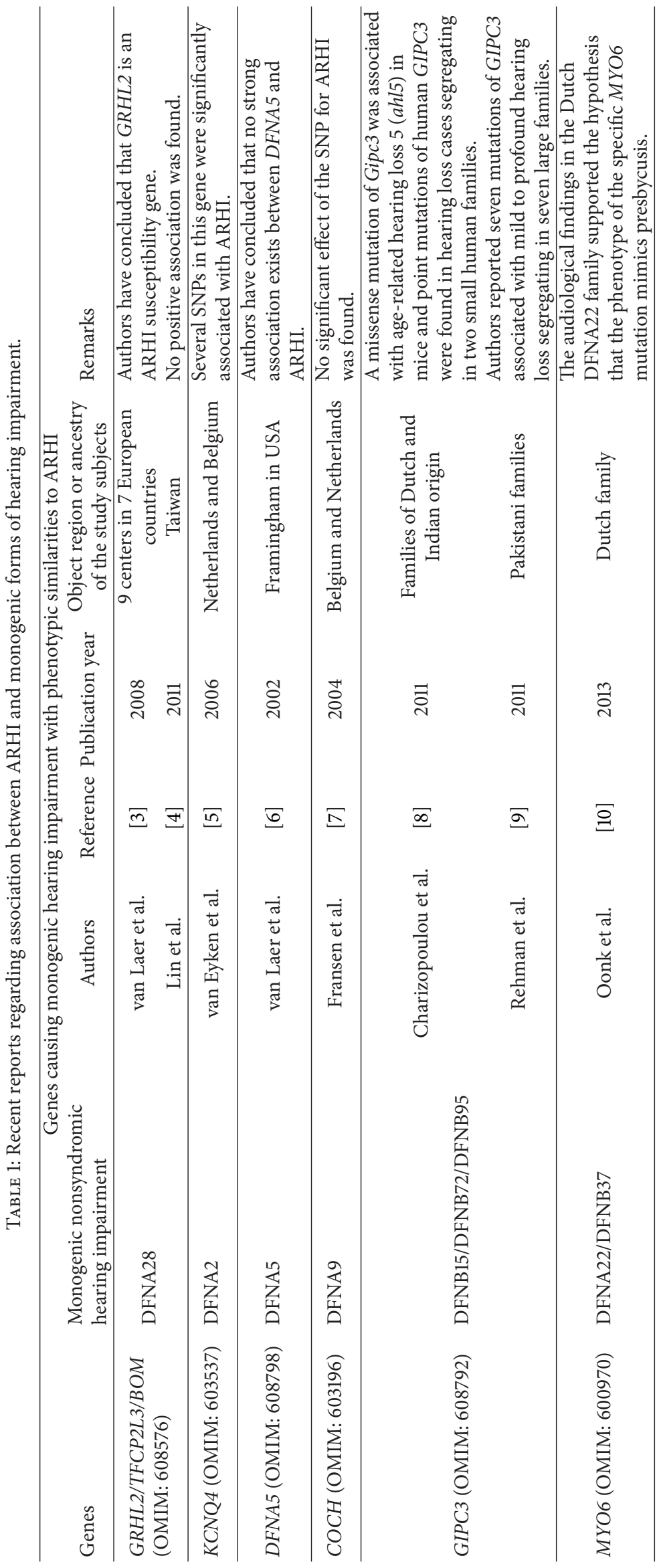


Apoptosis is a key contributor to the development of presbycusis or ARHI. In a review article focusing on the programmed death of the hair cell, Op de Beeck et al. discussed DFNA5 as one of several mutations in apoptosis genes that cause monogenic deafness [23]. They suggested that apoptosis contributes not only to the pathology of acquired forms of hearing impairment, but also to genetic hearing impairment.

2.4. DFNA9 = COCH (OMIM: 603196). The autosomal dominant nonsyndromic hearing disorder DFNA9 is characterized by late onset bilateral progressive high-frequency sensorineural hearing impairment with associated variable vestibular dysfunction and starts to manifest between 20 and 50 years of age [24]. Its features are similar to those of ARHI but hearing deterioration with DFNA9 occurs at a younger age. Point mutations in the COCH (cochlin or coagulation factor Chomology) gene, encoding the protein product, cochlin, are responsible for DFNA9-associated hearing loss. $\mathrm{COCH}$ mutations have been found in individuals living on four continents and the possibility that they play an important role in presbycusis and disorders of balance has been considered [25]. Phenotypic comparisons among families with different DFNA9-causing mutations in the $\mathrm{COCH}$ gene have been reported, with individuals presenting in the 95th percentile of the air conduction threshold levels for presbycusis for a given age and sex according to ISO $7029[24,26]$. In a study on the association between $\mathrm{COCH}$-variant T352S (rs 1045644) and ARHI, no significant effect of the SNP on quantitative trait values for ARHI was found [7].

2.5. DFNB15/DFNB72/DFNB95 = GIPC3 (OMIM: 608792). Autosomal recessive deafness 15 (DFNB15), also known as DFNB72 or DFNB95, is caused by homozygous mutations in the GIPC3 (GAIP interacting protein 3, C terminus) gene on chromosome 19p13.3.

Charizopoulou et al. identified a sequence polymorphism in the PDZ domain of Gipc3 as the cause of progressive sensorineural hearing loss (age-related hearing loss 5, ahl5) and audiogenic seizure susceptibility (jams1) in BLSW mice and they screened panels of families of Dutch and Indian origin presenting with autosomal recessive nonsyndromic hearing impairment to examine whether mutations in GIPC3 underlie human sensorineural hearing loss [8]. They identified the ahl5/jams1 and DFNB15/DFNB95 loci and revealed a function for Gipc3 in peripheral auditory signal transmission in mice and humans. They proposed that GIPC3 and its paralogs are excellent candidate genes for ARHI.

Susceptibility to metabotropic glutamate receptor 7 (mGluR7) or glutamate metabotropic receptor 7 (GRM7) excitotoxicity has been suggested as a risk factor for ARHI [27-29]. Charizopoulou et al. [8] noted that mGluR7 and Gipc3 show identical localization domains in the mouse cochlea and also share functional similarities, quoting studies of Friedman et al. [27] and Yi et al. [30]. Rehman et al. described one frameshift and six missense mutations in GIPC3 that cosegregate with DFNB72 associated with mild to profound hearing loss in six large families; their data provide statistically significant evidence for genetic linkage [9].

2.6. DFNA22/DFNB37 = MYO6 (OMIM: 600970). MYO6 encodes myosin VI, an unconventional actin-based motor protein, expressed in the hair cells of the inner ear that is necessary for maintaining their normal stereociliar structure $[31,32]$. MYO6 has been reported to be responsible for hearing loss associated with loci DFNA22 and DFNB37 [33, 34].

Oonk et al. compared the detailed audiometric characteristics of a large Dutch family with autosomal dominant hearing loss caused by a mutation in MYO6 with those of three different DFNA22 families published previously [10]. Hearing loss in the family closely resembled presbycusis and the authors hypothesized that rare variants of MYO6 may contribute to presbycusis.

\section{Genes Involved in Oxidative Stress, Biologic Stress Responses, and Mitochondrial Dysfunction}

Oxidative stress plays a major role in the overall aging process. Reactive oxygen species (ROS), such as superoxide anion, hydrogen peroxide, and hydroxyl radicals, are generated as byproducts of mitochondrial oxidative phosphorylation. Overproduction of free radicals and ROS results in damage to mitochondrial DNA (mtDNA). The mitochondrial "vicious cycle" theory of aging proposes that accumulation of mtDNA mutations may lead to enhanced mitochondrial ROS production and subsequent increased oxidative stress during aging [35].

Free radicals, ROS, and associated mitochondrial dysfunction are causes of age-related neurosensory hearing loss. Complex enzymatic and nonenzymatic systems act mutually to counteract ROS-mediated oxidative damage. Oxidative stress in the inner ear, secondary to the decrease in defense mechanisms caused by certain polymorphisms related to a battery of human antioxidant systems, could make individuals more susceptible to ARHI. A number of researchers have focused on several genes and loci involved in oxidative stress and have identified mitochondrial DNA mutations as candidate genes for the development of ARHI [36].

Two classes of antioxidant enzymes are active in the cochlea: enzymes involved in glutathione (GSH) metabolism (e.g., glutathione S-transferase, GST; glutathione peroxidase, GPX1; and glutathione reductase, GSR) and enzymes involved in the breakdown of superoxide anions and hydrogen peroxide (e.g., catalase, CAT, and $\mathrm{Cu} / \mathrm{Zn}$ superoxide dismutase, SOD1) [37-40].

3.1. NAT2 (OMIM: 612182), GST Family Genes. N-Acetyltransferase enzymes are responsible for the detoxification of large numbers of exogenic substrates and are important for balance of oxidative status. Two N-acetyltransferase isozymes, NAT1 and NAT2, have been identified in humans. A consensus nomenclature for the $\mathrm{N}$-acetyltransferase genes has been established, with $N A T 1^{*} 4$ and $N A T 2^{*} 4$ designated 
as the reference alleles for NAT1 (N-acetyltransferase 1) and NAT2 (N-acetyltransferase 2), respectively [41, 42]. An international nomenclature committee publishes updates in NAT1 and NAT2 alleles at the website http://nat.mbg.duth.gr. Variant NAT2 alleles or haplotypes possessing combinations of SNPs are segregated into clusters possessing a signature SNP either alone or in combination with others. Common human NAT2 alleles (haplotypes) discussed in the previous studies regarding ARHI are listed (see Table 2).

Ünal et al. investigated associations between ARHI and the NAT variations $N A T 2^{*} 5 A, N A T 2^{*} 6 A, N A T 2^{*} 7 A / B$, and NAT2 ${ }^{*} 14 A$ [44]. They studied a population of 68 white individuals of Turkish descent with presbycusis and found that the risk for presbycusis was 15.2-fold higher among individuals with the mutant allele $N A T 2^{*} 6 A$ than among those with a wild-type genotype $(P=.013)$.

The human glutathione S-transferase (GST) family is composed of at least 8 classes (alpha, A; kappa, K; mu, M; omega, O; Pi, P; sigma, S; theta, T; and zeta, Z) with multiple subfamilies per class $[45,46]$. GST enzymes play a key role in antioxidant defenses and a high level of GST enzymes is important to protect DNA from damage. Some classes, such as GSTM1 (mu, chromosome 1p13.3), GSTP1 (pi, chromosome 11q13), and GSTT1 (theta, chromosome 22q11.2), are polymorphic. The GSTT1 polymorphism is caused by deletion of a substantial part of the gene, causing a reduction of GSTT1 activity in all tissues, whereas in individuals homozygous for the GSTM1 deletion the enzyme is absent [45].

Angeli et al. investigated the association among audiometric patterns and polymorphisms of the antioxidant enzymes GSTT1, GSTM1, and NAT2 in a hospital-based cohort study of adults with ARHI [37]. Participants with mutant alleles for GSTT1 were more likely to have a "highfrequency steeply sloping" audiogram than participants with the wild-type genotype, suggesting that the basal turn of the cochlea is susceptible to GSTT1-regulated oxidative stress.

van Eyken et al. investigated an association between ARHI and genes related to oxidative stress, including polymorphisms in single genes (GSTM1, GSTT1, NAT2 $5 A$, $N A T 2^{*} 6 A$, and $N A T 2^{*} 7 A$ ) with a large set of 2,111 independent samples from a general European population and a Finnish population [43]. They observed significant associations between ARHI and NAT2* $6 \mathrm{~A}$ in the European population but no association between GSTM1 or GSTT1. However, in the Finnish population they found significant associations between ARHI and both genes.

Bared et al. investigated the association of different polymorphisms of GSTM1, GSTT1, or NAT2 with ARHI in 55 affected adults and 79 controls with normal hearing and showed an increased risk for ARHI among white adults carrying the GSTM1 and the GSTT1 null genotypes and the $N A T^{*} 6 A$ mutant allele [38]. Genotyping of the NAT2 polymorphisms $\left(N A T 25^{*} A, N A T 2^{*} 6 A, N A T 2^{*} 6 B, N A T 2^{*} 7 A\right.$, $N A T 2^{*} 7 B, N A T 2^{*} 14 A$, and $N A T 2^{*} 14 B$ ) was performed. The frequency of the $N A T 2^{*} 6 A$ mutant genotype (heterozygous and null genotype combined) was more frequent among patients with presbycusis (60\%) than in controls $(34 \%$; $P=0.0086, \mathrm{OR}=2.88,95 \%$ confidence interval $[95 \%$ $\mathrm{CI}]=1.355-6.141)$. The GSTT1 null genotype was present in
$34 \%$ of controls and in $60 \%$ of white patients with ARHI $(P=0.0067, \mathrm{OR}=2.843,95 \% \mathrm{CI}=1.379-5.860)$. The GSTM1 null genotype was more frequent in patients with ARHI.

3.2. mtDNA Mutations. Wallace and Fan have divided the mtDNA variants clinically relevant to disease susceptibility into three classes: recent deleterious mutations resulting in maternally transmitted disease, ancient adaptive variants that predispose individuals to disease in different environments, and the age-related accumulation of somatic mtDNA mutations that erode function and provide the aging clock [47]. These mutations can be inherited (constitutional) or acquired (somatic) and show a particularly poor genotype-phenotype correlation. Different mutations in the same gene causing different phenotypes and the same mutation at different levels of heteroplasmy causing different phenotypes result in the extraordinary clinical variability of mtDNA-associated diseases.

mtDNA mutations can exist as point mutations, nucleotide insertions or deletions, and large deletions. mtDNA deletions were reported to accumulate with age in a variety of tissues [48]. Analyses of human temporal bones indicated that the mtDNA 4977-bp deletion, the so-called "common" deletion, occurred frequently in ARHI patients $[49,50]$. Markaryan et al. used a real-time polymerase chain reaction assay to quantify the mtDNA in archival cochlear tissue samples derived from temporal bone and found a significant association between the common deletion level in human cochlear tissue and the severity of hearing loss [51].

mtDNA polymorphisms typically reflect different ethnic backgrounds. Specific mtDNA polymorphisms have now been classified into a number of mitochondrial haplogroups. Estimated worldwide haplotype frequencies (Mitomap: http://www.mitomap.org/) show that there are 20 recognized mtDNA haplogroups within the European community, 18 in the Asian community and 10 in the African community. Some of these haplotype groups have been associated with ARHI. Manwaring et al. found that haplogroups U and $\mathrm{K}$ associated independently with a higher prevalence of ARHI [52]. However, recently, Bonneux et al. found no significant associations of inherited common and rare coding variants, their mutation load, or European haplogroups with ARHI. They concluded that inherited mitochondrial variants are not a major factor in ARHI [53].

\section{Candidate Genes for Senescence, Other Geriatric Diseases, and Neurodegeneration}

4.1. APOE (OMIM: 107741). The APOE (Apolipoprotein E) gene encodes apolipoprotein $\mathrm{E}$ (ApoE), a multifunctional protein of the lipid and lipoprotein transport system involved mainly in metabolism of dietary lipids. The three major isoforms, human ApoE2, -E3, and -E4, are encoded by three common alleles of $A P O E$ called the $\varepsilon 2, \varepsilon 3$, and $\varepsilon 4$ alleles. These alleles represent haplotypes of two SNPs in the coding region of $A P O E$. The $\varepsilon 2$ allele encodes a cysteine (Cys) at amino acid position 112 and at position 158. Allele $\varepsilon 3$ encodes a Cys at 112 and an arginine (Arg) at 158, while the $\varepsilon 4$ allele 
TABLE 2: Common human NAT2 alleles (haplotypes) discussed in the previous studies regarding ARHI (modified from Hein, 2006 [41]).

\begin{tabular}{|c|c|c|c|c|}
\hline Allele (haplotype) $^{1}$ & Nucleotide change $(s)^{2}$ & Amino acid change $(\mathrm{s})^{3}$ & Catalytic activity $^{4}$ & Results in the previous ARHI studies \\
\hline$N A T 2^{*} 4$ & None & None & High & Wild type \\
\hline$N A T 2^{*} 5 A$ & $\underline{341 \mathrm{~T}>\mathrm{C}(\mathrm{rs} 1801280) ;} 481 \mathrm{C}>\mathrm{T}$ & $\mathrm{I} 114 \mathrm{~T}$ & Low & $\begin{array}{l}\text { Negative by Angeli et al. [37], van } \\
\text { Eyken et al. [43], Ünal et al. [44], and } \\
\text { Bared et al. [38] }\end{array}$ \\
\hline \multirow{2}{*}{$N A T 2^{*} 6 A$} & \multirow{2}{*}{$282 \mathrm{C}>\mathrm{T} ; 590 \mathrm{G}>\mathrm{A}(\mathrm{rs} 1799930)$} & \multirow{2}{*}{ R197Q } & \multirow{2}{*}{ Low } & Negative by Angeli et al. [37] \\
\hline & & & & $\begin{array}{l}\text { Positive by Ünal et al. [44], van Eyken } \\
\text { et al. [43], Bared et al. [38] }\end{array}$ \\
\hline$N A T 2^{*} 6 B$ & $590 \mathrm{G}>\mathrm{A}(\mathrm{rs1799930)}$ & R197Q & Low & $\begin{array}{l}\text { Negative by Angeli et al. [37] and } \\
\text { Bared et al. [38] }\end{array}$ \\
\hline \multirow{2}{*}{$N A T 2^{*} 7 A$} & \multirow{2}{*}{$\underline{857 \mathrm{G}>\mathrm{A}}$} & \multirow{2}{*}{ G286E } & \multirow{2}{*}{ Low } & Negative by van Eyken et al. [43] \\
\hline & & & & $\begin{array}{l}\text { (NAT2* } 7 A / B \text { combined) Negative by } \\
\text { Ünal et al. [44] }\end{array}$ \\
\hline$N A T 2^{*} 7 B$ & $282 \mathrm{C}>\mathrm{T} ; 857 \mathrm{G}>\mathrm{A}(\mathrm{rs1799931)}$ & G286E & Low & Negative by Bared et al. [38] \\
\hline \multirow[t]{2}{*}{$N A T 2^{*} 14 A$} & \multirow[t]{2}{*}{$191 \mathrm{G}>\mathrm{A}(\mathrm{rs} 1801279)$} & \multirow[t]{2}{*}{ R64Q } & \multirow[t]{2}{*}{ Low } & $\begin{array}{l}\text { Negative by Ünal et al. [44], Bared et } \\
\text { al. [38] }\end{array}$ \\
\hline & & & & $\begin{array}{l}\text { Data not shown by van Eyken et al. } \\
\text { [43] }\end{array}$ \\
\hline NAT2* $14 B$ & $191 \mathrm{G}>\mathrm{A}(\mathrm{rs} 1801279) ; 282 \mathrm{C}>\mathrm{T}$ & R64Q & Low & Negative by Bared et al. [38] \\
\hline
\end{tabular}

${ }^{1}$ Common NAT2 alleles (haplotypes) associated with low catalytic activity and slow acetylator phenotype are bolded.

${ }^{2}$ Signature SNP for each cluster is shown with underline.

${ }^{3}$ Amino-acid substitutions that confer reduced NAT2 activities.

Individuals homozygous for alleles indicated by boldface in annotation 1 are slow acetylators.

encodes an Arg at 112 and at 158 [54]. These polymorphisms within the $A P O E$ gene were strongly associated with lateonset Alzheimer disease. The $\varepsilon 4$ allele is the ancestral and high-risk form, the $\varepsilon 3$ allele is the most common in humans and the neutral allele, and the $\varepsilon 2$ allele is associated with decreased risk for Alzheimer disease.

O'Grady et al. found that, compared with the general population, the APOE $\varepsilon 4$ allele was less prevalent in a study population of 89 individuals (median age; 64 years) with nonsyndromic adult-onset sensorineural hearing loss, although they originally assumed that the $\varepsilon 4$ allele might predispose individuals to sensorineural hearing loss and ischemic injury in the cochlea by causing atherosclerotic vascular disease [55]. Recently, a converse finding that the presence of the APOE $\varepsilon 4$ allele was associated with ARHI overall frequency was demonstrated in the Leiden 85-plus study, a population-based study involving 85-year-olds [56]. The APOE $\varepsilon 4$ allele was associated with a 2.0 -fold increased risk for hearing impairment (95\% CI, 1.0-4.0).

4.2. MTHFR (OMIM: 607093). Folate metabolism is essential for cellular functioning. Polymorphisms in genes encoding critical enzymes in folate metabolism play important and interrelated roles in the pathophysiology of numerous disorders such as cancer and cardiocerebrovascular disease. The MTHFR (methylenetetrahydrofolate reductase) C677T polymorphism (rs1801133) is one of the most extensively investigated functional polymorphisms. It results in an alanine-to-valine substitution and a 30\% decrease in MTHFR enzyme activity in heterozygotes and a $60 \%$ decrease in homozygotes [57].
The MTHFR $\mathrm{T}$ allele has been shown to be associated with an increased risk for sudden sensorineural hearing loss in several different studies [58-60]. Interestingly, an inverse association between the same variant and ARHI was also demonstrated and may be due to a protective effect in individuals carrying the variant with a sufficient dietary supply of folate $[61,62]$. We investigated the effects of the methionine synthase (MTR) A2756G and MTHFR C677T gene polymorphisms on the risk for hearing impairment in community-dwelling Japanese adults aged 40-84 years who participated in the National Institute for Longevity Sciences, Longitudinal Study of Aging (NILS-LSA), an ongoing population-based biennial study. Cumulative data (5,167 samples in the accumulated total) were analyzed with generalized estimating equations. The MTHFR 677T allele was significantly associated with a reduced risk for hearing impairment only when the participants were wildtype homozygotes for MTR A2756G (per-T allele OR of MTHFR for development hearing impairment in the MTR AA genotype: 0.7609 [95\% CI: 0.6178-0.9372]). In addition, a subgroup analysis demonstrated that the favorable effect of the MTHFR 677T allele on the risk of developing hearing impairment was independent of serum folate and plasma total homocysteine levels. We speculated that the MTHFR $677 \mathrm{~T}$ allele has a potentially advantageous effect of preventing imbalances in the nucleotide pool during DNA synthesis, ensuring that DNA replication occurs with high fidelity.

4.3. EDN1 (OMIM: 131240). Endothelin-1, which is known to be an extremely potent vasoconstrictor peptide, is a prominent endothelial mediator. It is widely distributed 
throughout the mammalian body in cardiovascular and noncardiovascular tissues, including the nervous system, and is also expressed in the spiral modiolar artery, endolymphatic sac, vestibule, stria vascularis, and spiral ganglion cells [63, 64].

We have reported an association between the Lys198Asn (G/T) polymorphism (rs5370) in the EDN1 (endothelin-1) gene and hearing impairment in the aforementioned NILSLSA participants aged 40 years to 79 years at baseline [65]. Mutant T-allele carriers were demonstrated to have a higher risk for hearing impairment than homozygotic carriers of the wild-type allele in several models that adjust for different combinations of confounding variables.

4.4. UCP2 (OMIM: 601693). Uncoupling proteins (UCPs) are a family of mitochondrial proton transporters and may be regulators of thermal control and energy metabolism. UCPs are thought to be activated by superoxide and considered to decrease generation of mitochondrial free radicals.

We reported a relationship between gene polymorphisms in UCP1 (uncoupling protein 1) and UCP2 (uncoupling protein 2) and hearing impairment in the aforementioned NILSLSA [66]. Data were analyzed to assess the effects of UCP1 A-3826G and UCP2 Ala55Val polymorphisms on hearing impairment. The UCP1 A3826G polymorphism did not exhibit any significant association with ARHI; however, the UCP2 Ala55Val polymorphism did show a significant association with hearing impairment under the dominant, recessive, and additive models $(P<0.05)$.

4.5. Neurotransmitter-Related Genes, GRM7 (OMIM: 604101) and GRM8 (OMIM: 601116). GRM7 (glutamate receptor, metabotropic, 7) is thought to be central to maintaining glutamate synaptic transmission and homeostasis in the mammalian cochlea at the synapses between hair cells and the dendrites of afferent auditory nerve fibers [28].

Friedman et al. identified and subsequently validated a significant risk association between the GRM7 locus and ARHI [27]. In a study of three independent sample groups identified on the basis of a pooling genome-wide association study (GWAS), three genetic factors were described: a single SNP (rs11928865) and two haplotypes (haplotype block 6 consisting of SNPs rs11928865 and rs9877154, and haplotype block 7 consisting of SNPs rs6804466, rs3828472, rs12497688, rs9819783, and rs11920109). The authors demonstrated that the protein product, mGluR7, was expressed in inner and outer hair cells and in the spiral ganglion nerve cell bodies of the inner ear of mice and humans. GRM7 mRNA expression was also present in the neurons of the spiral ganglion in the mouse cochlea.

Newman et al. have delved further into the effect of GRM7 on ARHI [28]. They performed mixed modeling analyses in a European-American population from Rochester in the United States to explore the relationship between GRM7 haplotypes (haplotype blocks 6 and 7) and SNP genotypes and various measures of auditory perception, such as the free-field hearing-in-noise test (HINT) and suprathreshold gap detection tasks. They showed that GRM7 alleles were associated primarily with peripheral measures of hearing loss and particularly with speech detection in older adults.

Luo et al. conducted a case-control candidate gene association study in an elderly male Han Chinese population composed of 982 men with ARHI and 324 controls with normal hearing [29]. The results of the study suggest that GRM7 SNP rs11928865 (TT genotype) occurs more frequently in ARHI patients with certain audiogram-shape subtypes of ARHI, namely, sloping-shaped and $2-4 \mathrm{kHz}$ abrupt lossshaped phenotype patterns.

New candidate loci were reported from a GWAS for hearing quantitative traits among participants recruited from several isolated villages within the international consortium called G-EAR [67]. Eight primary loci were detected in a series of genes expressed within the inner ear, including GRM8 (glutamate receptor, metabotropic, 8), and the candidate genes located in positive GWAS regions belonged to several different gene families, which overlap only insensibly with those already identified as causing hearing impairments.

4.6. ESRRG (OMIM: 602969). Estrogen has recently been identified as a novel modulator of hearing function $[68,69]$. There is now considerable evidence linking estrogen signaling, the estrogen receptors, and estrogen-related receptors (ESRR) with auditory protection $[68,70]$.

Nolan et al. conducted an investigation involving a total of 6134 individuals in three independent cohorts: (i) the 1958 British birth cohort; (ii) a London ARHL case-control cohort; and (iii) a cohort from isolated populations of Italy and Silk Road countries to evaluate whether variants in estrogen signaling genes may be risk factors for adult-onset hearing loss. The rs2818964 SNP in the ESRRG (estrogen-related receptor, gamma) gene was associated with hearing status in the London ARHL cohort, the minor allele being associated with poorer hearing but only in women. This association was replicated in the Carlantino cohort and the combined cohort of isolated populations from Italy and Silk Road countries, both in the direction of the allelic effect and in the femalespecific association [71]. In line with these findings, ESRRG was shown to be expressed in the adult mouse inner ear and hearing assessment in Esrrg knock-out mice revealed worse impairment in female mice $(-/-)$ than in male mice $(-/-)$. These results indicate that ESRRG plays a role in maintenance of hearing in both humans and mice.

\section{Future Prospects}

The degree of ARHI varies substantially among individuals, as is also observed in senescence of other functions. It is not always true that deterioration of hearing with age is inevitable. During the last 10 years or so, tremendous progress has been made through human genetic research into hearing impairment and our understanding of the complex mechanisms of hearing has increased greatly. It might be that certain environmental risk factors are potentially detrimental in a limited number of individuals only, depending on their genetic background. It is hopeful that successful preventive intervention customized according to knowledge of genetic 
susceptibility will help to preserve good hearing even in the oldest old.

\section{Conflict of Interests}

The authors declare that there is no conflict of interests regarding the publication of this paper.

\section{Acknowledgment}

This study was supported in part by the Research Grant for Longevity Sciences (25-2) from the Ministry of Health, Labour and Welfare of Japan.

\section{References}

[1] Y. Uchida, S. Sugiura, F. Ando, T. Nakashima, and H. Shimokata, "Molecular genetic epidemiology of age-related hearing impairment," Auris Nasus Larynx, vol. 38, no. 6, pp. 657-665, 2011.

[2] A. M. D. Martini, D. Stephens, and A. P. Read, Genes, Hearing, and Deafness : From Molecular Biology to Clinical Practice, Informa Healthcare, London, UK, 2007.

[3] L. van Laer, E. van Eyken, E. Fransen et al., "The grainyhead like 2 gene (GRHL2), alias TFCP2L3, is associated with age-related hearing impairment," Human Molecular Genetics, vol. 17, no. 2, pp. 159-169, 2008.

[4] Y. Lin, C. Wu, C. Hsu, J. Hwang, and T. Liu, "The grainyheadlike 2 gene (GRHL2) single nucleotide polymorphism is not associated with age-related hearing impairment in Han Chinese," Laryngoscope, vol. 121, no. 6, pp. 1303-1307, 2011.

[5] E. van Eyken, L. van Laer, E. Fransen et al., "KCNQ4: a gene for age-related hearing impairment?” Human Mutation, vol. 27, no. 10, pp. 1007-1016, 2006.

[6] L. van Laer, E. van Eyken, E. Fransen et al., "The grainyhead like 2 gene (GRHL2), alias TFCP2L3, is associated with age-related hearing impairment," Human Molecular Genetics, vol. 17, no. 2, pp. 159-169, 2008.

[7] E. Fransen, L. Van Laer, N. Lemkens et al., "A Novel Zscore-based method to analyze candidate genes for age-related hearing impairment," Ear and Hearing, vol. 25, no. 2, pp. 133141, 2004.

[8] N. Charizopoulou, A. Lelli, M. Schraders et al., "Gipc3 mutations associated with audiogenic seizures and sensorineural hearing loss in mouse and human," Nature communications, vol. 2, p. 201, 2011.

[9] A. U. Rehman, K. Gul, R. J. Morell et al., "Mutations of GIPC3 cause nonsyndromic hearing loss DFNB72 but not DFNB81 that also maps to chromosome 19p," Human Genetics, vol. 130, no. 6, pp. 759-765, 2011.

[10] A. M. M. Oonk, J. M. Leijendeckers, E. M. Lammers et al., "Progressive hereditary hearing impairment caused by a MYO6 mutation resembles presbyacusis," Hearing Research, vol. 299, pp. 88-98, 2013.

[11] M. Werth, K. Walentin, A. Aue et al., "The transcription factor grainyhead-like 2 regulates the molecular composition of the epithelial apical junctional complex," Development, vol. 137, no. 22, pp. 3835-3845, 2010.

[12] L. M. Peters, D. W. Anderson, A. J. Griffith et al., "Mutation of a transcription factor, TFCP2L3, causes progressive autosomal dominant hearing loss, DFNA28," Human Molecular Genetics, vol. 11, no. 23, pp. 2877-2885, 2002.
[13] B. Vona, I. Nanda, C. Neuner, T. Müller, and T. Haaf, "Confirmation of GRHL2 as the gene for the DFNA28 locus," American Journal of Medical Genetics A, vol. 161, no. 8, pp. 2060-2065, 2013.

[14] C. Kubisch, B. C. Schroeder, T. Friedrich et al., "KCNQ4, a novel potassium channel expressed in sensory outer hair cells, is mutated in dominant deafness," Cell, vol. 96, no. 3, pp. 437-446, 1999.

[15] T. Kharkovets, J. Hardelin, S. Safieddine et al., "KCNQ4, a K channel mutated in a form of dominant deafness, is expressed in the inner ear and the central auditory pathway," Proceedings of the National Academy of Sciences of the United States of America, vol. 97, no. 8, pp. 4333-4338, 2000.

[16] L. M. Dominguez and K. M. Dodson, "Genetics of hearing loss: focus on DFNA2," Application of Clinical Genetics, vol. 5, pp. 97104, 2012.

[17] P. J. Coucke, P. van Hauwe, P. M. Kelley et al., "Mutations in the KCNQ4 gene are responsible for autosomal dominant deafness in four DFNA2 families," Human Molecular Genetics, vol. 8, no. 7, pp. 1321-1328, 1999.

[18] T. Naito, S. Nishio, Y. Iwasa et al., "Comprehensive genetic screening of KCNQ4 in a large autosomal dominant nonsyndromic hearing loss cohort: genotype-phenotype correlations and a founder mutation," PLOS ONE, vol. 8, no. 5, Article ID e63231, 2013.

[19] L. van Laer, E. H. Huizing, M. Verstreken et al., "Nonsyndromic hearing impairment is associated with a mutation in DFNA5," Nature Genetics, vol. 20, no. 2, pp. 194-197, 1998.

[20] A. B. Cheng, D. Y. Han, P. Dai et al., "A novel DFNA5 mutation, IVS8+4 A>G, in the splice donor site of intron 8 causes lateonset non-syndromic hearing loss in a Chinese family," Clinical Genetics, vol. 72, no. 5, pp. 471-477, 2007.

[21] H. Park, H. Cho, J. Baek et al., "Evidence for a founder mutation causing DFNA5 hearing loss in East Asians," Journal of Human Genetics, vol. 55, no. 1, pp. 59-62, 2010.

[22] K. O. De Beeck, G. Van Camp, S. Thys et al., "The DFNA5 gene, responsible for hearing loss and involved in cancer, encodes a novel apoptosis-inducing protein," European Journal of Human Genetics, vol. 19, no. 9, pp. 965-973, 2011.

[23] K. Op de Beeck, J. Schacht, and G. Van Camp, "Apoptosis in acquired and genetic hearing impairment: The programmed death of the hair cell," Hearing Research, vol. 281, no. 1-2, pp. 18-27, 2011.

[24] R. J. Pauw, P. L. M. Huygen, G. M. Colditz, and C. W. R. J. Cremers, "Phenotype analysis of an Australian DFNA9 family with the I109N COCH mutation," Annals of Otology, Rhinology and Laryngology, vol. 120, no. 6, pp. 414-421, 2011.

[25] N. G. Robertson, C. W. R. J. Cremers, P. L. M. Huygen et al., "Cochlin immunostaining of inner ear pathologic deposits and proteomic analysis in DFNA9 deafness and vestibular dysfunction," Human Molecular Genetics, vol. 15, no. 7, pp. 1071$1085,2006$.

[26] S. International Organization for, Acoustics: threshold of hearing by air conduction as a function of age and sex for otologically normal persons. (International Organization for Standardization, Geneva, Switzerland, 1984).

[27] R. A. Friedman, L. van Laer, M. J. Huentelman et al., "GRM7 variants confer susceptibility to age-related hearing impairment," Human Molecular Genetics, vol. 18, no. 4, pp. 785-796, 2009.

[28] D. L. Newman, L. M. Fisher, J. Ohmen et al., "GRM7 variants associated with age-related hearing loss based on auditory 
perception," Hearing Research, vol. 294, no. 1-2, pp. 125-132, 2012.

[29] H. Luo, T. Yang, X. Jin et al., "Association of GRM7 variants with different phenotype patterns of age-related hearing impairment in an elderly male Han Chinese population," PLoS ONE, vol. 8, no. 10, Article ID e77153, 2013.

[30] Z. Yi, R. S. Petralia, Z. Fu et al., "The role of the PDZ protein GIPC in regulating NMDA receptor trafficking," Journal of Neuroscience, vol. 27, no. 43, pp. 11663-11675, 2007.

[31] N. Hilgert, V. Topsakal, J. van Dinther, E. Offeciers, P. van de Heyning, and G. van Camp, "A splice-site mutation and overexpression of MYO6 cause a similar phenotype in two families with autosomal dominant hearing loss," European Journal of Human Genetics, vol. 16, no. 5, pp. 593-602, 2008.

[32] V. Topsakal, N. Hilgert, J. van Dinther et al., "Genotype-phenotype correlation for dfna22: characterization of non-syndromic, autosomal dominant, progressive sensorineural hearing loss due to MYO6 mutations," Audiology and Neurotology, vol. 15, no. 4, pp. 211-220, 2010.

[33] S. Melchionda, N. Ahituv, L. Bisceglia et al., "MYO6, the human homologue of the gene responsible for deafness in Snell's waltzer mice, is mutated in autosomal dominant nonsyndromic hearing loss," The American Journal of Human Genetics, vol. 69, no. 3, pp. 635-640, 2001.

[34] Z. M. Ahmed, R. J. Morell, S. Riazuddin et al., "Mutations of MYO6 are associated with recessive deafness, DFNB37," The American Journal of Human Genetics, vol. 72, no. 5, pp. 13151322, 2003.

[35] I. Afanas'ev, "Signaling and damaging functions of free radicals in aging-free radical theory, hormesis, and TOR," Aging and Disease, vol. 1, no. 2, pp. 75-88, 2010.

[36] T. Yamasoba, F. R. Lin, S. Someya, A. Kashio, T. Sakamoto, and K. Kondo, "Current concepts in age-related hearing loss: epidemiology and mechanistic pathways," Hearing Research, vol. 303, pp. 30-38, 2013.

[37] S. I. Angeli, A. Bared, X. Ouyang, L. L. Du, D. Yan, and X. Z. Liu, "Audioprofiles and antioxidant enzyme genotypes in presbycusis," Laryngoscope, vol. 122, no. 11, pp. 2539-2542, 2012.

[38] A. Bared, X. Ouyang, S. Angeli et al., "Antioxidant enzymes, presbycusis, and ethnic variability," Otolaryngology-Head and Neck Surgery, vol. 143, no. 2, pp. 263-268, 2010.

[39] R. Kopke, K. A. Allen, D. Henderson, M. Hoffer, D. Frenz, and T. van de Water, "A radical demise. Toxins and trauma share common pathways in hair cell death," Annals of the New York Academy of Sciences, vol. 884, pp. 171-191, 1999.

[40] P. I. Carlsson, L. van Laer, E. Borg et al., "The influence of genetic variation in oxidative stress genes on human noise susceptibility," Hearing Research, vol. 202, no. 1-2, pp. 87-96, 2005.

[41] D. W. Hein, "N-acetyltransferase 2 genetic polymorphism: effects of carcinogen and haplotype on urinary bladder cancer risk," Oncogene, vol. 25, no. 11, pp. 1649-1658, 2006.

[42] D. W. Hein, "N-acetyltransferase SNPs: emerging concepts serve as a paradigm for understanding complexities of personalized medicine," Expert Opinion on Drug Metabolism and Toxicology, vol. 5, no. 4, pp. 353-366, 2009.

[43] E. van Eyken, G. van Camp, E. Fransen et al., "Contribution of the $\mathrm{N}$-acetyltransferase 2 polymorphism NAT2 $* 6 \mathrm{~A}$ to agerelated hearing impairment," Journal of Medical Genetics, vol. 44, no. 9, pp. 570-578, 2007.

[44] M. Ünal, L. Tamer, Z. N. Doğruer, H. Yildirim, Y. Vayisoğlu, and H. Çamdeviren, "N-acetyltransferase 2 gene polymorphism and presbycusis," Laryngoscope, vol. 115, no. 12, pp. 2238-2241, 2005.
[45] D. L. Eaton and T. K. Bammler, "Concise review of the glutathione S-transferases and their significance to toxicology," Toxicological Sciences, vol. 49, no. 2, pp. 156-164, 1999.

[46] E. L. Abel, S. M. Opp, C. L. M. J. Verlinde, T. K. Bammler, and D. L. Eaton, "Characterization of atrazine biotransformation by human and murine glutathione S-transferases," Toxicological Sciences, vol. 80, no. 2, pp. 230-238, 2004.

[47] D. C. Wallace and W. Fan, "Energetics, epigenetics, mitochondrial genetics," Mitochondrion, vol. 10, no. 1, pp. 12-31, 2010.

[48] G. A. Cortopassi and N. Arnheim, "Detection of a specific mitochondrial DNA deletion in tissues of older humans," Nucleic Acids Research, vol. 18, no. 23, pp. 6927-6933, 1990.

[49] U. Bai, M. D. Seidman, R. Hinojosa, and W. S. Quirk, "Mitochondrial DNA deletions associated with aging and possibly presbycusis: a human archival temporal bone study," The American Journal of Otology, vol. 18, no. 4, pp. 449-453, 1997.

[50] P. Dai, W. Yang, S. Jiang et al., "Correlation of cochlear blood supply with mitochondrial DNA common deletion in presbyacusis," Acta Oto-Laryngologica, vol. 124, no. 2, pp. 130-136, 2004.

[51] A. Markaryan, E. G. Nelson, and R. Hinojosa, "Quantification of the mitochondrial DNA common deletion in presbycusis," Laryngoscope, vol. 119, no. 6, pp. 1184-1189, 2009.

[52] N. Manwaring, M. M. Jones, J. W. Jie et al., "Mitochondrial DNA haplogroups and age-related hearing loss," Archives of Otolaryngology-Head and Neck Surgery, vol. 133, no. 9, pp. 929-933, 2007.

[53] S. Bonneux, E. Fransen, E. Van Eyken et al., "Inherited mitochondrial variants are not a major cause of age-related hearing impairment in the European population," Mitochondrion, vol. 11, no. 5, pp. 729-734, 2011.

[54] G. D. Schellenberg and T. J. Montine, "The genetics and neuropathology of Alzheimer's disease," Acta Neuropathologica, vol. 124, no. 3, pp. 305-323, 2012.

[55] G. O'Grady, A. L. Boyles, M. Speer, F. DeRuyter, W. Strittmatter, and G. Worley, "Apolipoprotein E alleles and sensorineural hearing loss," International Journal of Audiology, vol. 46, no. 4, pp. 183-186, 2007.

[56] C. Kurniawan, R. G. J. Westendorp, A. J. M. de Craen, J. Gussekloo, J. de Laat, and E. van Exel, "Gene dose of apolipoprotein E and age-related hearing loss," Neurobiology of Aging, vol. 33, no. 9, 2012.

[57] R. Rozen, "Genetic predisposition to hyperhomocysteinemia: Deficiency of methylenetetrahydrofolate reductase (MTHFR)," Thrombosis and Haemostasis, vol. 78, no. 1, pp. 523-526, 1997.

[58] P. Capaccio, F. Ottaviani, V. Cuccarini et al., "Sudden hearing loss and MTHFR 677C>T/1298A>C gene polymorphisms," Genetics in Medicine, vol. 7, no. 3, pp. 206-208, 2005.

[59] Y. Uchida, S. Sugiura, F. Ando, H. Shimokata, and T. Nakashima, "Association of the C677T polymorphism in the methylenetetrahydrofolate reductase gene with sudden sensorineural hearing loss," Laryngoscope, vol. 120, no. 4, pp. 791-795, 2010.

[60] M. Fusconi, A. Chistolini, N. Angelosanto et al., "Role of genetic and acquired prothrombotic risk factors in genesis of Sudden sensorineural hearing loss," Audiology and Neurotology, vol. 16, no. 3, pp. 185-190, 2011.

[61] J. Durga, L. J. C. Anteunis, E. G. Schouten, M. L. Bots, F. J. Kok, and P. Verhoef, "Association of folate with hearing is dependent on the 5,10- methylenetetrahdyrofolate reductase $677 \mathrm{C} \rightarrow \mathrm{T}$ mutation," Neurobiology of Aging, vol. 27, no. 3, pp. 482-489, 2006. 
[62] Y. Uchida, S. Sugiura, F. Ando, T. Nakashima, and H. Shimokata, "Hearing impairment risk and interaction of folate metabolism related gene polymorphisms in an aging study," BMC Medical Genetics, vol. 12, article 35, 2011.

[63] D.-Y. Xu, Y.-D. Tang, S.-X. Liu, and J. Liu, "Distribution and significance of endothelin 1 in guinea pig cochlear lateral wall," Journal of Laryngology and Otology, vol. 121, no. 8, pp. 721-724, 2007.

[64] D. Xu, Y. Tang, S. Liu, and J. Liu, "Expression and significance of endothelin 1 in spiral ganglion cells of guinea pig," International Journal of Pediatric Otorhinolaryngology, vol. 72, no. 2, pp. 189192, 2008.

[65] Y. Uchida, S. Sugiura, T. Nakashima, F. Ando, and H. Shimokata, "Endothelin-1 gene polymorphism and hearing impairment in elderly Japanese," Laryngoscope, vol. 119, no. 5, pp. 938-943, 2009.

[66] S. Sugiura, Y. Uchida, T. Nakashima, F. Ando, and H. Shimokata, "The association between gene polymorphisms in uncoupling proteins and hearing impairment in Japanese elderly," Acta OtoLaryngologica, vol. 130, no. 4, pp. 487-492, 2010.

[67] G. Girotto, N. Pirastu, R. Sorice et al., "Hearing function and thresholds: A genome-wide association study in European isolated populations identifies new loci and pathways," Journal of Medical Genetics, vol. 48, no. 6, pp. 369-374, 2011.

[68] J. S. McCullar and E. C. Oesterle, "Cellular targets of estrogen signaling in regeneration of inner ear sensory epithelia," Hearing Research, vol. 252, no. 1-2, pp. 61-70, 2009.

[69] L. A. Tremere, R. F. Kovaleski, K. Burrows, J. K. Jeong, and R. Pinaud, "Mechanistic basis and functional roles of long-term plasticity in auditory neurons induced by a brain-generated estrogen," The Journal of Neuroscience, vol. 32, no. 46, pp. 1647816495, 2012.

[70] M. Hultcrantz, R. Simonoska, and A. E. Stenberg, "Estrogen and hearing: a summary of recent investigations," Acta OtoLaryngologica, vol. 126, no. 1, pp. 10-14, 2006.

[71] L. S. Nolan, H. Maier, I. Hermans-Borgmeyer et al., "Estrogenrelated receptor gamma and hearing function: evidence of a role in humans and mice," Neurobiology of Aging, vol. 34, no. 8, pp. 2077.el-2077.e9, 2013. 


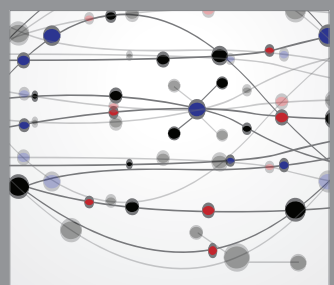

The Scientific World Journal
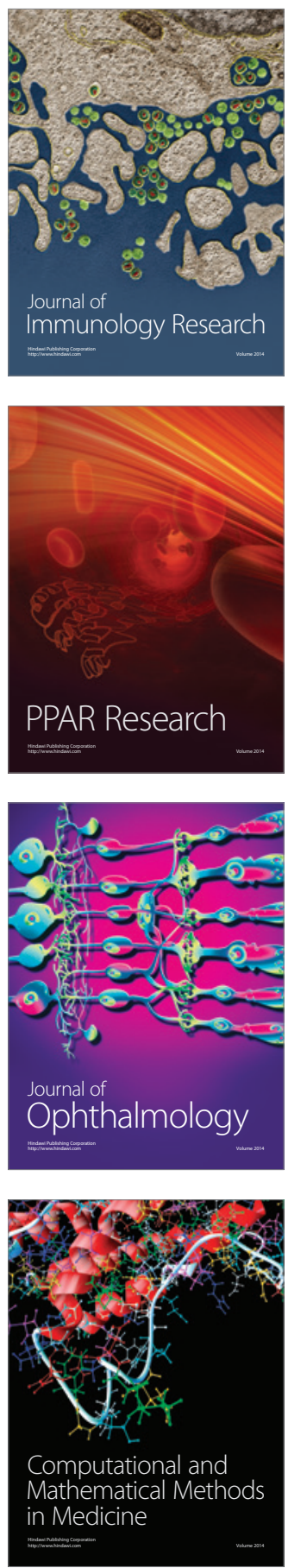

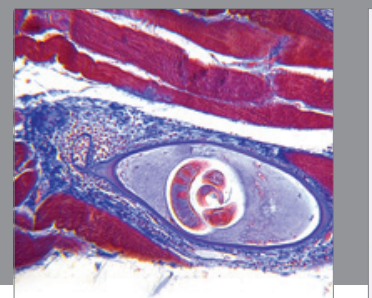

Gastroenterology

Research and Practice
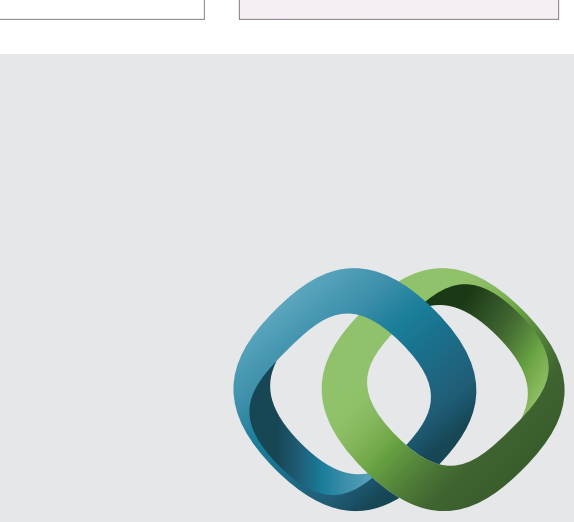

\section{Hindawi}

Submit your manuscripts at

http://www.hindawi.com
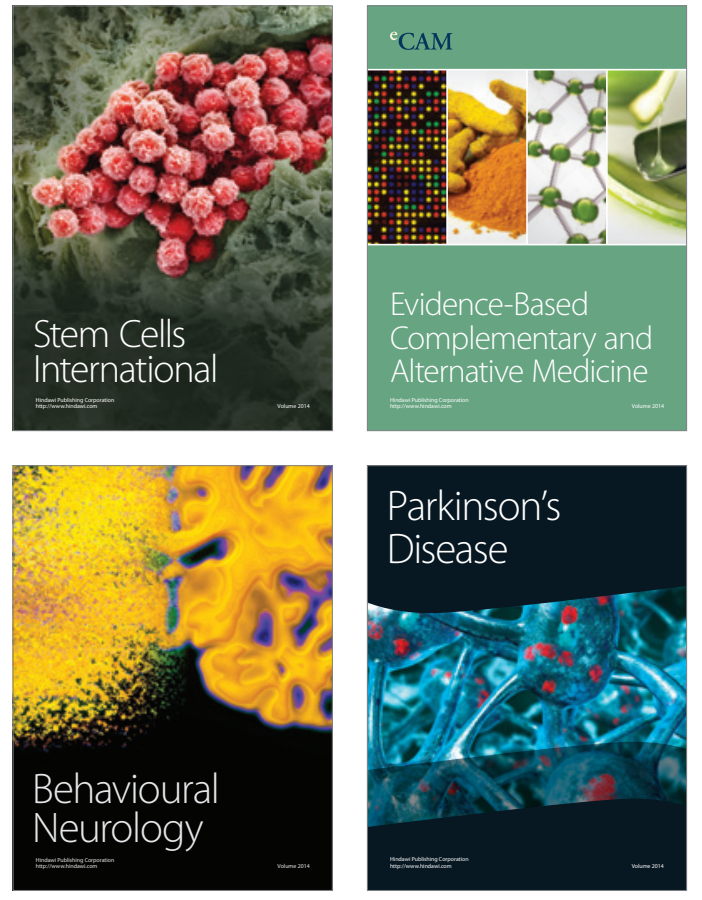
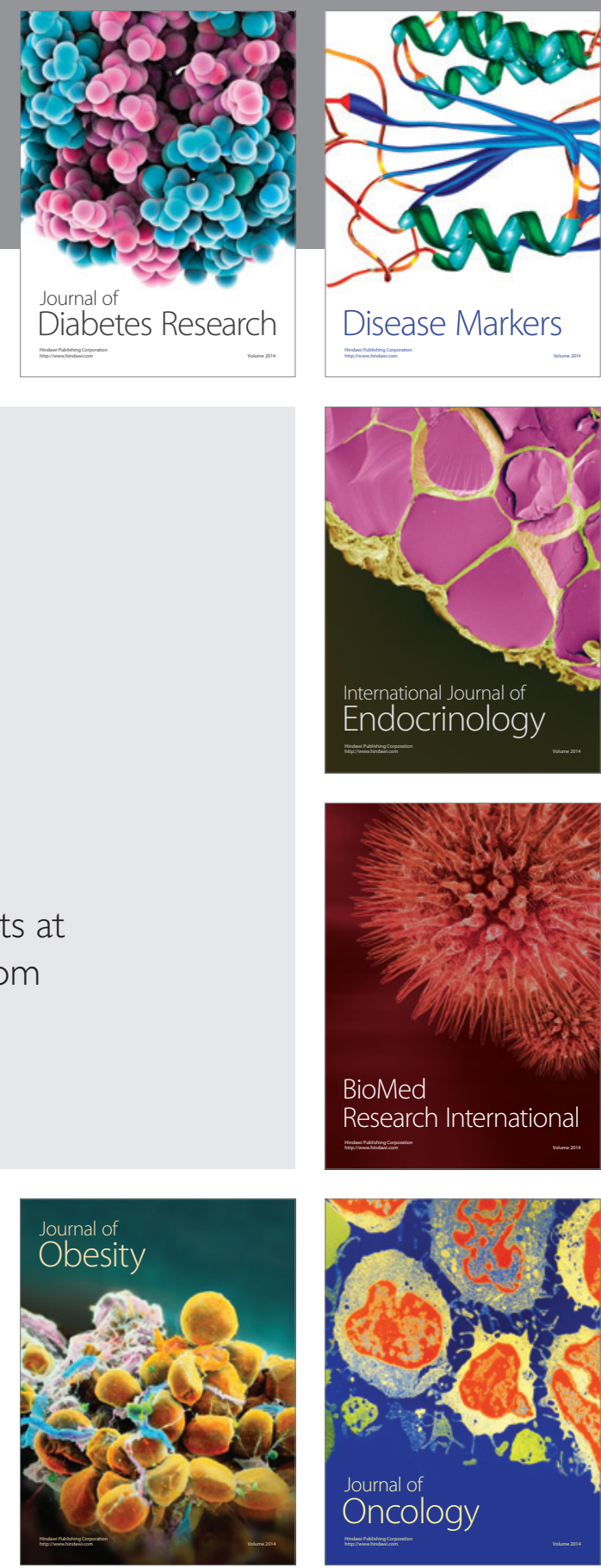

Disease Markers
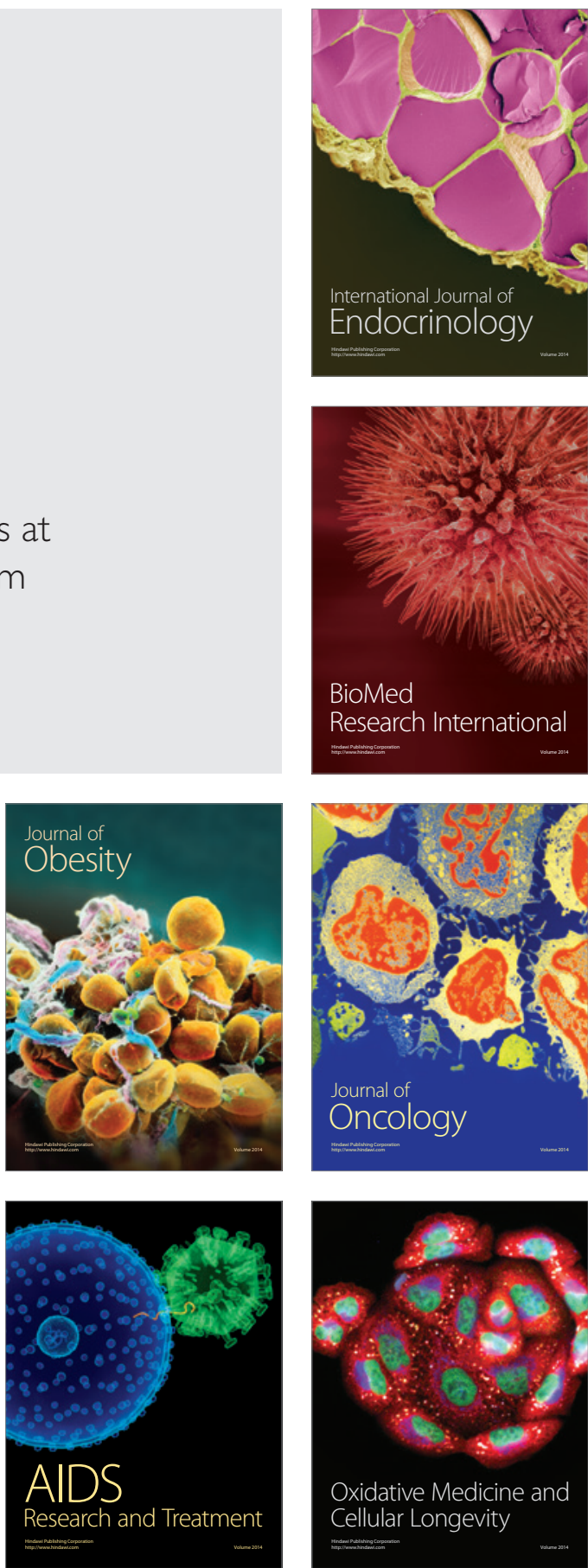